\title{
Duplicity Among $\lambda$ Boo Stars: the New Case of HD 204041
}

\author{
M. Gerbaldi ${ }^{1}$ and R. Faraggiana ${ }^{2}$ \\ ${ }^{1}$ Institut d'Astrophysique de Paris and Université de Paris-Sud XI, Orsay, France \\ email: gerbaldi@iap.fr \\ ${ }^{2}$ Dipartimento di Astronomia,Università degli Studi di Trieste, Italy \\ email: faraggiana@oats.inaf.it
}

\begin{abstract}
The spectrum of the classical $\lambda$ Boo star HD 204041 is revealed to be a composite one.
\end{abstract}

Keywords. stars: atmospheres, stars: binaries: spectroscopic, stars: individual (HD 204041)

\section{Introduction}

The $\lambda$ Boo stars are a fascinating class of stars: it is the only class of A-type stars with abundances lower than solar, in spite of the fact that they have the kinematic and photometric properties of Pop I stars. The most plausible theory (at present) to interpret this peculiar behavior is based on the diffusion-accretion model proposed by Venn \& Lambert (1990). The key point of this model is the existence of a surrounding disk of dust and gas which would be the remnant of the star formation material and from which the - metal-depleted - gas is accreted, the metals being incorporated into the dust.

The large variety of objects classified as $\lambda$ Boo and the lack of any relation between their physical parameters and the measured abundance anomalies, coupled with the recent detection of bright companions near several $\lambda$ Boo candidates prompted us to search the objects that have composite spectra: a combination of two similar spectra which can be confused with that of a single metal-poor star when analyzed by adopting their average $\mathrm{T}_{\text {eff }}$ and $\log \mathrm{g}$ parameters (Faraggiana \& Bonifacio 1999). The detection of duplicity is tricky due to their mean-high $\mathrm{v} \sin \mathrm{i}$, which produces broad and shallow lines. We have developed a strategy to detect such composite spectra (Faraggiana et al 2004), and apply this analysis to one more object: HD 204041, a "well known" $\lambda$ Boo star.

\section{Data and observations}

HD 204041 has been considered as a classical $\lambda$ Boo since its first classification by Gray \& Garrison (1987) and since then it is included in the lists of well-established $\lambda$ Boo stars (Gray 1988, 1997; Paunzen 1997, 2001).

Properties that make HD 204041 a good candidate for the class of undetected binaries are, first, found in the literature. They concern the large dispersion of the measurements of fundamental parameters such as: radial velocity, v sin $\mathrm{i}, \mathrm{T}_{\text {eff }}$ and $\log \mathrm{g}$ (Table 1) and the abundances of the elements $\mathrm{C}, \mathrm{N}, \mathrm{O}$, and $\mathrm{S}$ which should be solar but vary from author to author and even, for the same author, from paper to paper. In Table 2 we give the relative abundances of $[\mathrm{C} / \mathrm{H}]$ and $[\mathrm{O} / \mathrm{H}]$ as determined up to now or adopted in the course of a spectroscopic analysis by several authors. 
Table 1. Published values of the radial velocity (RV), v sin i, $\mathrm{T}_{\text {eff }}$ and $\log \mathrm{g}$ for HD 204041

\begin{tabular}{|c|c|c|}
\hline parameter & value & reference \\
\hline RV & $-9 \mathrm{kms}^{-1}$ & GCRV (1953), BSC, Simbad \\
\hline RV & $-18.20 \pm 37.19 \mathrm{kms}^{-1}$ & $\begin{array}{l}\text { (internal error } 0.46 \mathrm{~km} / \mathrm{s} \text { ) Grenier et al. (1999) } \\
\text { conclude that the star is a suspected double. }\end{array}$ \\
\hline $\mathrm{v} \sin \mathrm{i}$ & $55 \mathrm{kms}^{-1}$ & Abt \& Morrell 1995, adopted by Gray (1997) \\
\hline $\mathrm{v} \sin \mathrm{i}$ & $65 \mathrm{kms}^{-1}$ & Stürenburg 1993, Paunzen et al. (2002) \\
\hline $\mathrm{v} \sin \mathrm{i}$ & $68 \pm 5 \mathrm{kms}^{-1}$ & Holweger \& Rentzsch-Holm 1995; Royer et al. (2002) \\
\hline $\mathrm{v} \sin \mathrm{i}$ & $70 \mathrm{kms}^{-1}$ & Gray \& Corbally (1993) \\
\hline $\mathrm{v} \sin \mathrm{i}$ & $75 \mathrm{kms}^{-1}$ & Gray (1988) \\
\hline $\mathrm{v} \sin \mathrm{i}$ & $85 \mathrm{kms}^{-1}$ & Dworetsky (1974) \\
\hline $\mathrm{T}_{\text {eff }}$ and $\log \mathrm{g}$ & 7909 and 3.58 & {$[\mathrm{Fe} / \mathrm{H}]=-1.69$ in Wilhelm et al. (1999) } \\
\hline $\mathrm{T}_{\text {eff }}$ and $\log \mathrm{g}$ & 7980 and 3.97 & Paunzen et al. (2002) \\
\hline $\mathrm{T}_{\text {eff }}$ and $\log \mathrm{g}$ & 8100 and 4.03 & Stürenburg (1993) \\
\hline $\mathrm{T}_{\text {eff }}$ and $\log \mathrm{g}$ & 8100 and 4.0 & Paunzen et al. (1999a); Kamp et al. (2001) \\
\hline $\mathrm{T}_{\text {eff }}$ and $\log \mathrm{g}$ & 8100 and 4.1 & Iliev \& Barzova (1995) \\
\hline $\mathrm{T}_{\text {eff }}$ and $\log \mathrm{g}$ & 8300 and 4.2 & Paunzen et al. (1999b) \\
\hline
\end{tabular}

Table 2. HD 204041 relative abundances for C and O

\begin{tabular}{crl}
\hline element & {$[\mathrm{X} / \mathrm{H}]$} & reference \\
\hline $\mathrm{C}$ & -0.30 & Stürenburg (1993) \\
$\mathrm{C}$ & -0.20 & Paunzen et al (1999b) \\
$\mathrm{C}$ & -0.58 & adopted from literature in Paunzen et al (2002) \\
$\mathrm{C}$ & -0.75 & Paunzen et al. (1999a) (NLTE value $=-0.81)$ \\
$\mathrm{O}$ & 0.00 & Paunzen et al. (1999a) (NLTE value $=-0.38)$ \\
$\mathrm{O}$ & -0.42 & adopted from literature in Paunzen et al (2002) \\
$\mathrm{O}$ & -0.50 & Paunzen et al (1999b) \\
\hline
\end{tabular}

We have collected optical spectra of HD 204041 at the ESO $(\mathrm{R}=28000)$, the Observatoire de Haute-Provence $(\mathrm{R}=20000)$ and the Observatoire du Pic du Midi $(\mathrm{R}=32000)$.

Figure 1 displays the observations obtained at the ESO with the Echelec spectrograph on Sept 5th, 1993 and Sept 18th, 1994. These data are normalized spectra with no correction for the radial velocity due to the Earth motion, but those corrections are very similar for each spectrum, the observational data being taken one year apart. The positions of the lines have changed and this is interpreted as due to the orbital motion of the star induced by duplicity.

These spectra are compared to a synthetic one, computed in LTE from Kurucz models (1993), in the region of $\mathrm{MgII}$, the red wing of $\mathrm{H} \beta$ and the core of $\mathrm{H} \alpha$. Among the several metal abundance analyses we choose that made by Stürenburg (1993) which includes the highest number of elements and we adopted his parameters and abundances to compute this synthetic spectrum.

Figures 2, 3, and 4 display the comparisons in these three spectral domains between the observations and the synthetic spectrum.

\section{Results and conclusion}

From Figures 1 to 4 and the previous analysis we note that:

- the line profiles vary from spectrum to spectrum in shape and position

- there are strange line profiles: flat core, asymmetry compared to the computed ones 


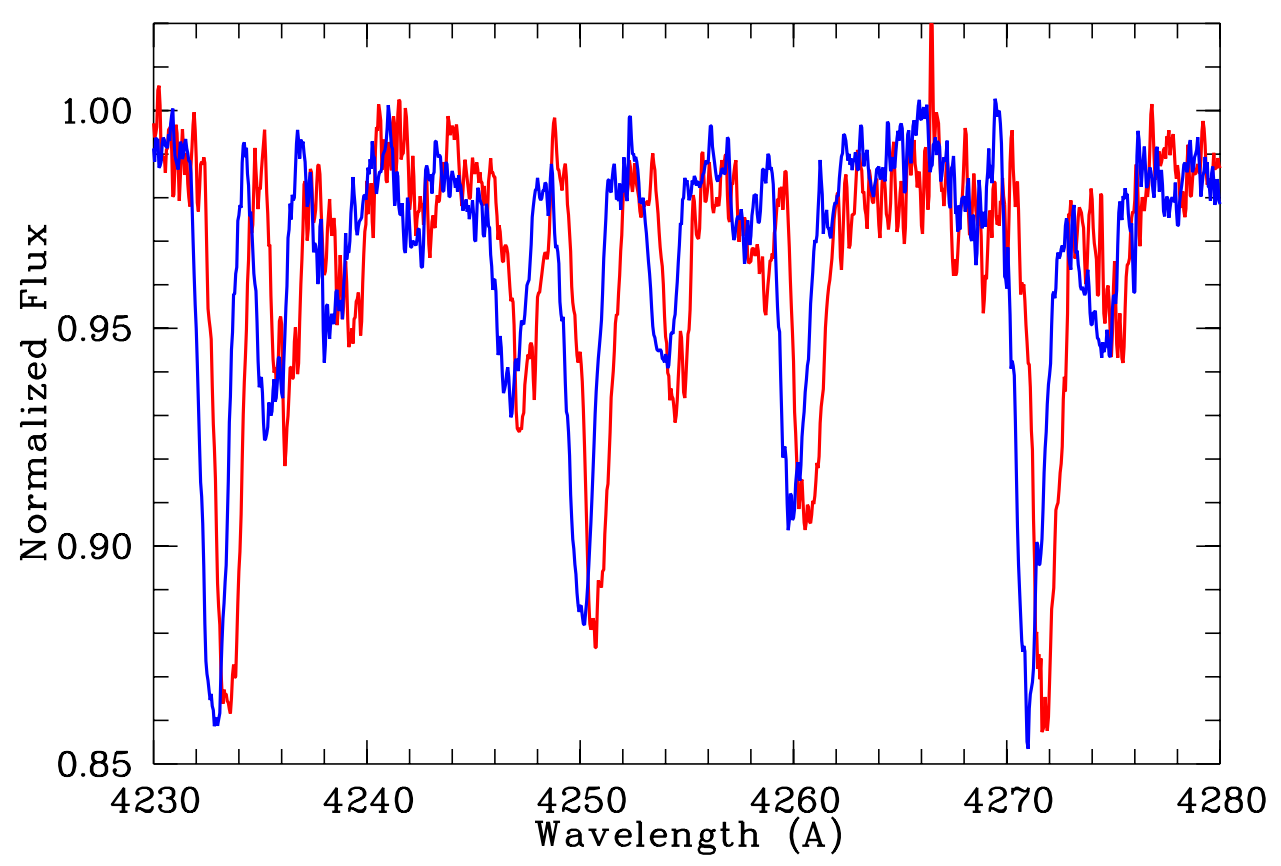

Figure 1. The 4250 A region observed on Sept 5th, 1993 (in red) compared to the same region observed on Sept 18th 1994 (in blue).
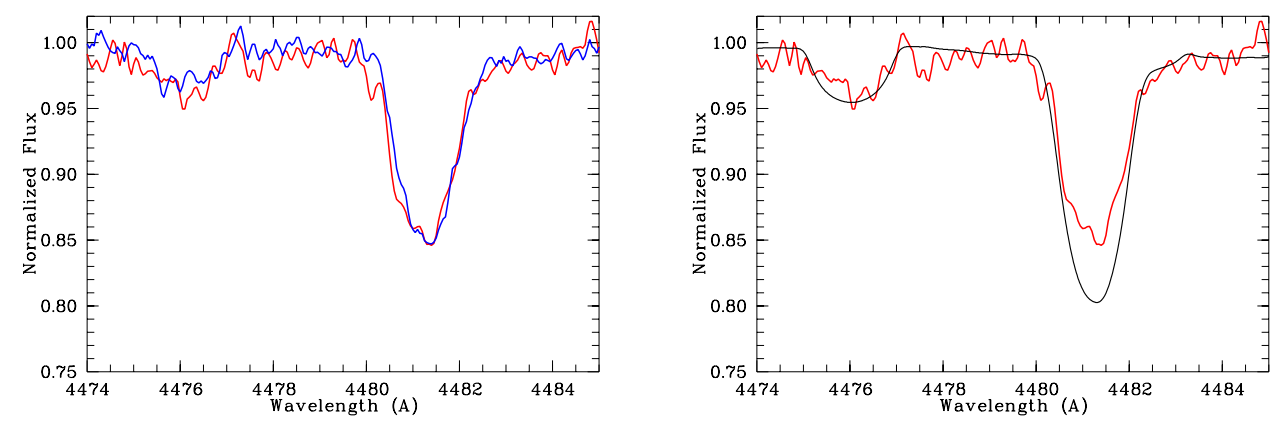

Figure 2. Profile of MgII doublet. On left side, in red: Sept 5th, 1993; in blue: Sept 18th 1994. On right side, in red: Sept 5th 1993; in black: the computed spectra.

- the core of $\mathrm{H} \alpha$ profile should be deeper than that of a synthetic spectra computed with LTE

- the presence of lines with different broadening in the same spectra

- there are inconsistent results with the abundance analysis attempted with synthetic spectra ie the ionization equilibrium is not respected

- the radial velocity is variable

- the abundances of $\mathrm{C}$ and $\mathrm{O}$ are not solar.

We conclude that HD 204041 is another $\lambda$ Boo star among the class of undetected binaries showing a composite spectrum. 


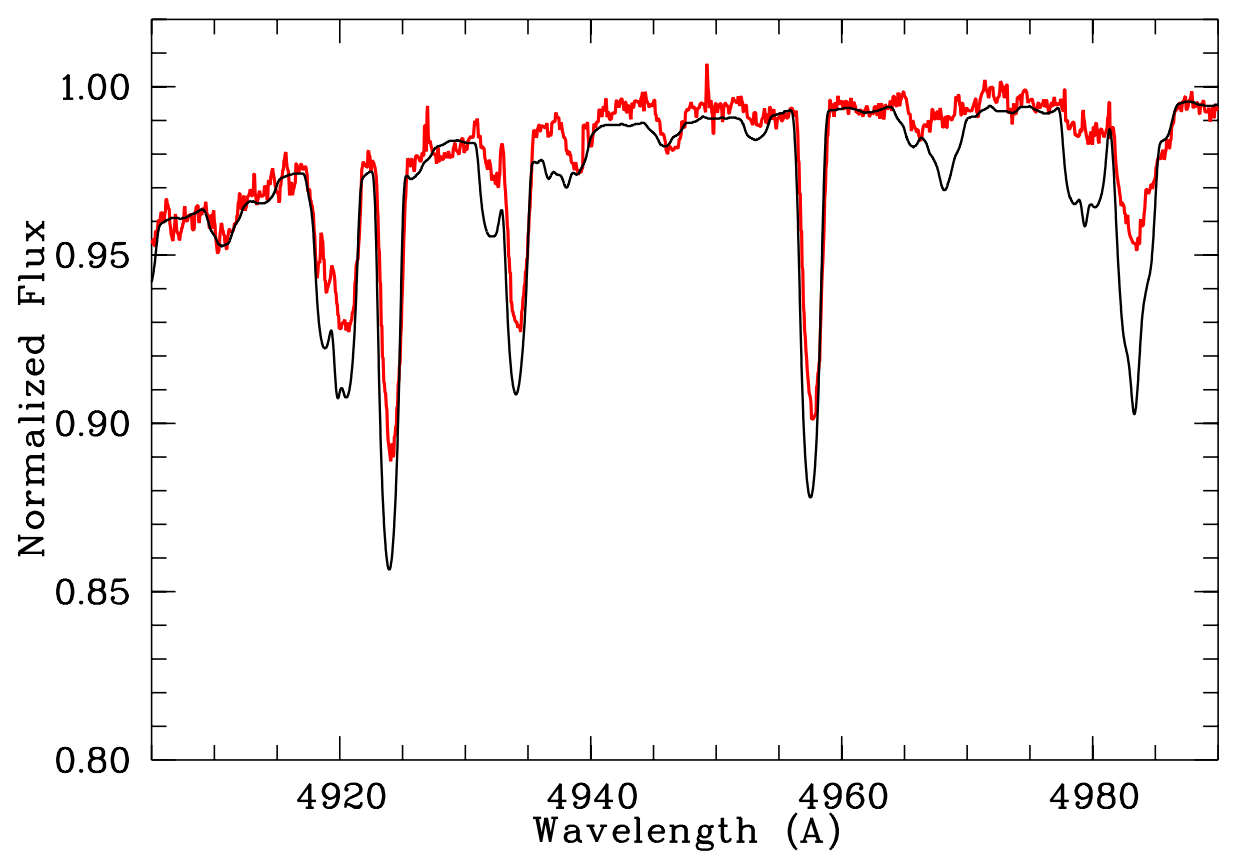

Figure 3. The observed red wing of $\mathrm{H} \beta$ compared to a synthetic spectrum (in red: observation on August 1st 1998; in black: the computed spectrum).

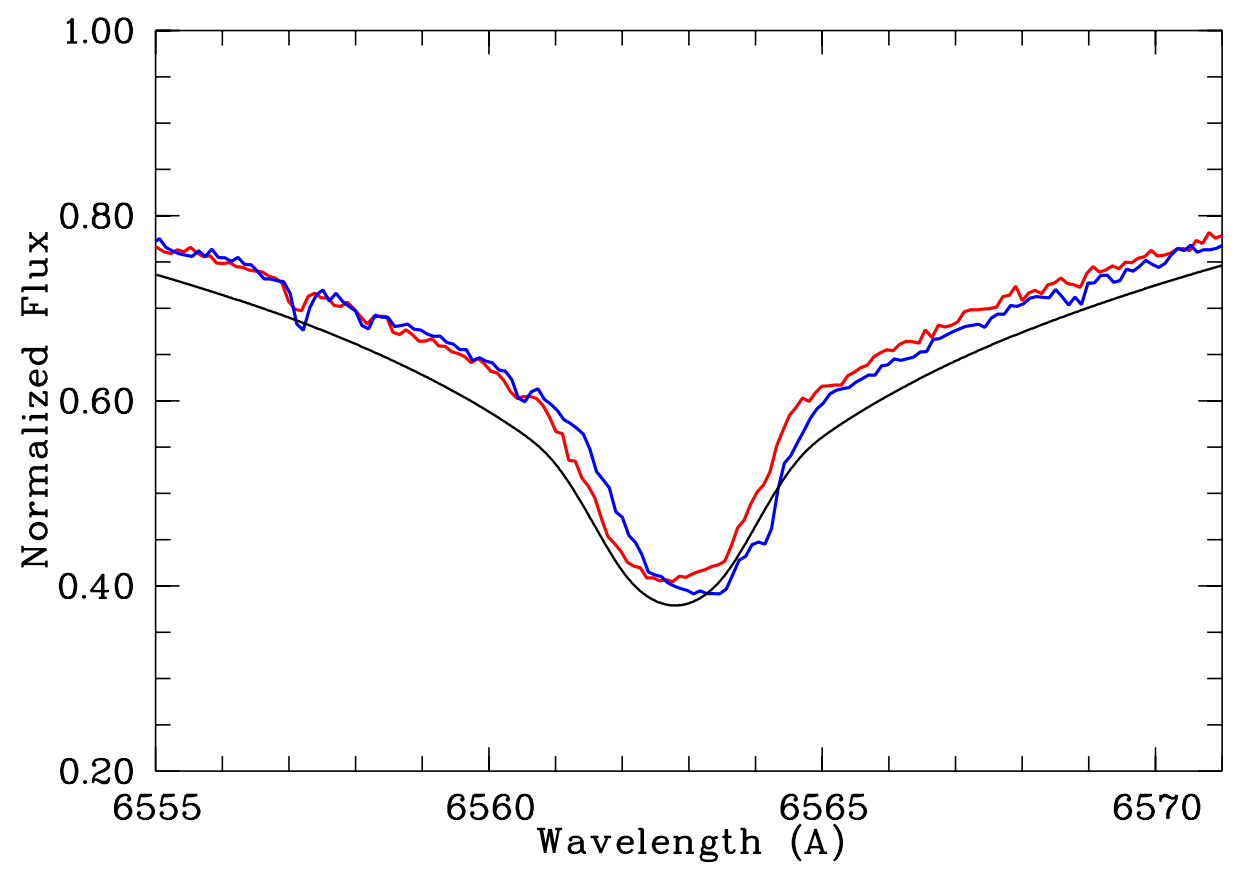

Figure 4. The core of $\mathrm{H} \alpha$ compared to a synthetic spectrum (in red : observation on Sept 29th 2000, in blue: observation on Oct 12th 2002, in black, smooth line: the computed spectrum.) 


\section{References}

Abt, H.A. \& Morrell, N. 1995 ApJS 99, 135

Dworetsky, M.M. 1974 ApJS 28, 101

Faraggiana, R. \& Bonifacio, P. 1999 A $\mathscr{E} A$ 349, 521

Faraggiana, R., Bonifacio, P., Caffau, E. et al. $2004 A \mathscr{E} A$ 425, 615

Gray, R.O. 1988 AJ 95, 220

Gray, R.O. 1997, 3rd Conf. Faint Blue Stars page 237

Gray, R.O. \& Garrison, R.F 1987, ApJS 65,581

Gray, R.O. \& Corbally, C.J. 1993 ApJ 106, 632

Grenier, S. et al. $1999 A \& A S$ 135, 503

Holweger, H. \& Rentzsch-Holm, I. 1995 A\& A 303, 819

Iliev, I.K. \& Barzova, I. 1995 A $\& A$ 302, 735

Kamp, I., Iliev, I.K., Paunzen, E. et al. $2001 A \& A$ 375, 899

Kurucz, R. 1993 CD-ROM No 13

Paunzen, E., Weiss, W.W., Heiter, U. \& North, P. 1997 A $\& A S$ 123, 93

Paunzen, E., Kamp, I., Iliev, I.K. et al. 1999a A\&A 345, 597

Paunzen, E., Andrievsky, S.M., Chernyshova, I.V. et al. 1999b A $\& A$ 351, 981

Paunzen, E. 2001 A $\& A$ 373, 633

Paunzen, E., Iliev, I. K., Kamp, I. \& Barzova, I. S. 2002 MNRAS 336, 1030

Royer, F., Gerbaldi, M., Faraggiana, R. et al. 2002 A\&A 381, 105

Stürenburg, S. 1993 A\&A 277, 139

Venn, K. A. \& Lambert, D.L. 1990 ApJ 363, 234

Wilhelm, R., Beers, T.C. \& Gray, R.O. 1999 AJ 117, 2308 